\title{
Sex Hormones and Gender Effects following Trauma-Hemorrhage
}

IRSHAD H. CHAUDRY • FARIBA MOEINPOUR • WEN-KE WANG • SHAOLONG YANG • RAGHAVAN RAJU • WILLIAM B. HUBBARD

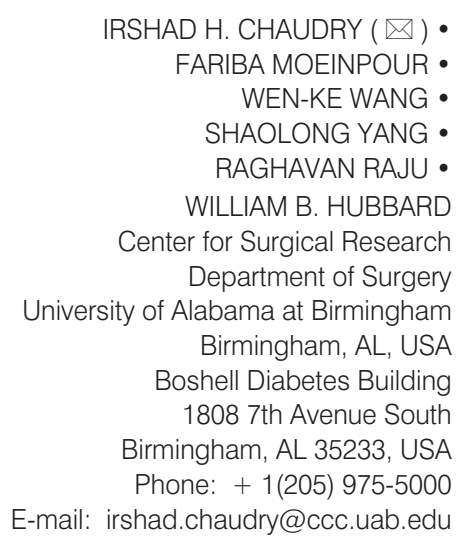

$\operatorname{IRSHAD~H.~CHAUDRY~}(\bowtie) \bullet$ FARIBA MOEINPOUR • WEN-KE WANG • SHAOLONG YANG • RAGHAVAN RAJU • WILLIAM B. HUBBARD Center for Surgical Research Department of Surgery University of Alabama at Birmingham Birmingham, AL, USA Boshell Diabetes Building 1808 7th Avenue South Birmingham, AL 35233, USA

Phone: + 1(205) 975-5000

E-mail: irshad.chaudry@ccc.uab.edu

\begin{abstract}
Trauma is the leading cause of death in the industrialized world between the ages of one and 40. A number of risk factors including age and gender have been implicated in this regard. It is therefore not surprising that the majority of trauma victims are young males. Their mortality rate following trauma is not only higher compared to females, but they are also more prone to subsequent sepsis. Age and gender are therefore important factors in the prevalence of traumatic injury as well as in susceptibility to subsequent septic complications.
\end{abstract}

Keywords: trauma, mortality, age, gender, sex hormones, sepsis, cardiovascular/immunological alterations

\section{Introduction}

The late Roger Bone noted a preponderance of morbidity and mortality in males compared to females in four major sepsis studies. $(1,2)$ Experimental studies have shown that following the induction of sepsis (as produced by cecal ligation and puncture), the survival rate in females is significantly higher than in age-matched males. (3) It should be noted that the survival advantage is only conferred in females in the proestrus cycle, i.e., cycle with high estrogen and progesterone levels. (3) Subsequent studies showed that even if the animals underwent traumahemorrhage and induction of subsequent sepsis, the survival in proestrus females was significantly higher than in age-matched males. (3) This advantage the proestrus females have in tolerance to trauma-hemorrhage and sepsis disappears post-menopausally or following ovariectomy. In this regard, the ovariectomized females were also found to be extremely susceptible to sepsis following trauma-hemorrhage and resuscitation as compared to females in the proestrus cycle. (4) Thus, our hypothesis was that the prevailing hormonal milieu at the time of injury (and not hrs or days later) dictates whether there will be immune and cardiovascular maintenance or depression of those parameters after injury. The results, in fact, indicate that proestrus females tolerate trauma-hemorrhage and sepsis better than males and females in the other estrus cycles.

\section{Sex Hormones and Gender Effects}

In attempting to understand why the proestrus females tolerate traumahemorrhage and sepsis better than males and ovariectomized females, studies examined cardiac index in males, estrus and proestrus females 24 hrs after trauma-hemorrhage and resuscitation. (5) The results indicated that cardiac index was depressed even 24 hrs following trauma-hemorrhage and resuscitation in males and estrus females, but not in proestrus females. (5) Likewise, hepatocellular function, which was measured by the clearance of indocyanine green, was also markedly depressed in males and estrus females following trauma-hemorrhage and resuscitation, but was found to be normal in proestrus females undergoing trauma-hemorrhage and resuscitation. (5)

Subsequent studies examined whether decreasing female sex steroid levels due to aging or ovariectomy would cause cardiovascular depression following trauma-hemorrhage, and if so, whether $17 \beta$-estradiol (E2) administration has any salutary effects, and whether the effects of E2 are mediated via estrogen receptors (ER). The results indicated that cardiac index was 
markedly decreased in ovariectomized females following trauma-hemorrhage; however, administration of a single dose of E2 following trauma-hemorrhage in ovariectomized animals normalized the depressed cardiac index. If, however, the estrogen receptor antagonist ICI 182,780 (ICl) was given along with E2, the cardiac index was depressed and was found to be the same as in vehicle-treated animals. (5) These results therefore suggest that administration of a single dose of E2 in ovariectomized animals restores the cardiac index following trauma-hemorrhage and resuscitation, and the effects of E2 are mediated via the ER, since blocking the ER by using $\mathrm{ICl}$ totally abolished the salutary effects of E2 on cardiac index. Similarly, hepatocellular function, which was depressed following trauma-hemorrhage and resuscitation, was also normalized by administration of a single dose of E2 following traumahemorrhage and resuscitation, (5) and as in the case of cardiac index, hepatocellular function was also depressed if $\mathrm{ICl}$ was given along with E2. (5)

\section{Cardiac function following trauma-hemorrhage and sex hormones}

Since cardiac function following trauma-hemorrhage was differentially affected in males and proestrus females, studies examined whether there was any correlation between cardiac function and cardiomyocyte intracellular IL-6 levels and/or IL-16 expression. Cardiomyocyte IL-6 mRNA expression and intracellular IL-6 production increased following trauma-hemorrhage. (5) Moreover, cardiac IL-6 protein levels increased after trauma-hemorrhage in freshly isolated heart. Furthermore, there was an inverse correlation between cardiomyocyte IL-6 levels and cardiac function following traumahemorrhage. (5) These results therefore suggest that the salutary effects of E2 on cardiac function following trauma-hemorrhage may be due, in part, to decreased IL-6 synthesis in cardiomyocytes. (5)

Studies also examined whether admini- stration of flutamide (androgen receptor (AR) antagonist) in male rats following trauma-hemorrhage influences cardiac function, and if it does whether it does so by blocking the AR or by modulating the ER expression. The results indicated that cardiac contractility $(+\mathrm{dP} /$ $\mathrm{dt}_{\text {max }}$ ) was significantly depressed in males following trauma-hemorrhage and resuscitation; however, administration of flutamide significantly improved the depressed myocardial contractility. (5) Of significance is the fact that ER- $\alpha$ and ER- $\beta$ protein expression in the myocardium were significantly depressed in males following trauma-hemorrhage and resuscitation; however, administration of flutamide normalized the expression of not only ER- $\alpha$ but also ER- $\beta$ protein expression. In contrast to $\mathrm{ER}$, there were no alterations in AR protein expression. These results therefore suggest that the mechanism of the salutary effects of flutamide on cardiac output following trauma-hemorrhage appears to be mediated via the upregulation of ER- $\alpha$ and ER- $\beta$ mRNA and protein expression.

The results also indicated that although splenocyte proliferative capacity (an index of immune function) was depressed in males following trauma-hemorrhage, it was found to be unaltered or enhanced in proestrus females under those conditions. Similarly, splenocyte IL-2 productive capacity was also markedly decreased in males but not in proestrus females following traumahemorrhage and resuscitation. $(3,4)$ This depression in splenocyte functions was correlated with a marked increase in plasma corticosteroid levels in males following trauma-hemorrhage; however, the corticosteroid levels in proestrus females were significantly higher than in age-matched males in shams but the levels decreased following trauma-hemorrhage and resuscitation. $(3,4)$ Thus, opposite responses in the plasma corticosteroid levels were observed between males and proestrus females following trauma-hemorrhage and resuscitation.

Since proestrus females had maintained or enhanced immune functions following trauma-hemorrhage compared to males, studies examined whether changing the hormonal milieu in females to mimic that of males would also cause immunosuppression following trauma-hemorrhage similar to males. The results indicated that female mice, which had slow-releasing $5 \boldsymbol{\alpha}$-dihydrotestosterone (DHT) pellets implanted, showed marked increase in plasma DHT levels; those levels were similar to corresponding age-matched males. Furthermore, these females with the DHT pellets had significantly lower plasma E2 levels, which were again similar to the age-matched males. Under these conditions, the splenocyte proliferative capacity which was increased or unaltered following trauma-hemorrhage in proestrus females was markedly decreased in females with DHT implantation, and the decrease in splenocyte proliferation was similar to age-matched males. $(3,4)$ Likewise, females with DHT implanted had decreased splenocyte proliferative capacity, which was again similar to the age-matched males.

Another question was whether androgens contribute to the immunosuppression that is observed following trauma and hemorrhage. For these studies, castration of the animals was carried out two weeks prior to traumahemorrhage to decrease DHT levels. The results indicated that splenocyte functions were markedly depressed in sham castrated animals; however, they were not decreased in animals which underwent castration two weeks prior to the onset of trauma-hemorrhage. $(3,4)$ Similarly, splenic macrophage capacity to release cytokines following trauma-hemorrhage was decreased in sham castrated animals but was not decreased in pre-castrated animals under those conditions. In additional studies, flutamide was administered following trauma-hemorrhage and resuscitation in males and the results showed that the depressed splenocyte proliferative capacity and IL-2 release capacity (which was observed in splenocytes following trauma-hemorrhage and resuscitation) were normalized by 
administration of flutamide following trauma-hemorrhage. Furthermore, the depressed macrophage functions were also improved by flutamide administration following trauma-hemorrhage. $(3,4)$ Additional studies indicated that the salutary effects of flutamide administration on splenic T Iymphocyte functions were associated with increased expression of ER in male T lymphocytes following flutamide administration. Flutamide administration following trauma-hemorrhage and resuscitation also decreased the susceptibility of animals to subsequent sepsis. $(4,5)$

Studies have also shown that administration of E2 in males following trauma-hemorrhage was effective in restoring the depressed splenocyte and macrophage functions following trauma-hemorrhage and resuscitation. $(3,4)$ Additional studies suggested that increased synthesis and decreased catabolism of DHT are the reason for loss of T lymphocyte functions and cardiovascular functions in males following trauma-hemorrhage and resuscitation as evidenced by decreased synthesis of IL-2 and depressed cardiac functions. $(5,6,7)$ The converse occurs in proestrus females, i.e., increased synthesis and decreased catabolism of E2 as the reason for the maintenance of $T$ lymphocyte and cardiovascular functions under those conditions. $(3,4)$ Additional results indicated that in addition to E2 and flutamide, the depressed immune and cardiovascular functions in males and ovariectomized females following trauma-hemorrhage can also be improved/normalized by administration of prolactin, dehydroepiandrosterone (DHEA), or 4-hydroxy androstenedione following trauma-hemorrhage. In addition, administration of three different doses of DHEA following trauma-hemorrhage also decreased the mortality from subsequent sepsis. $(3,4)$ In addition to experimental studies, epidemiological studies have suggested that the physiological pattern of pre-menopausal adult female sex hormones provide a survival advantage. (6) A recent prospective study from Germany indicated that the incidence of multiple organ dysfunction and failure after severe trauma was significantly greater in young males than in females, and was also the same with regard to proinflammatory cytokines. (7) Another prospective study involving over 4,000 patients showed that hormonally active women tolerate shock-trauma better than males. (8) Although studies have also reported a lack of gender difference in the outcome following sepsis, the differences in the results may be due to the genetically diverse nature of patient sets analyzed, significant variation in post-injury care at different centers, preexisting co-morbidities, different injury severity and hormonal milieu of female patients at the time of injury (which was not measured). (9) Thus, hormonal milieu immediately after injury should be measured in clinical studies of trauma.

Studies have also shown that the salu- tary effects of E2 on cardiac function are mediated by ER- $\beta$ and ER- $\beta$-induced upregulation of several heat shock proteins. (3) Furthermore, ER- $\alpha$ as well as ER- $\beta$ have also been shown to have salutary effects on organ functions following trauma-hemorrhage. (3) An additional study has shown that administration of E2 $(1 \mathrm{mg} / \mathrm{kg}$ BW in a very small volume) after removal of $60 \%$ of the circulating blood volume permitted the animal to remain in a state of permissive hypotension even in the absence of any additional fluid administration for 3 hrs. (10) The results showed that survival in the E2-treated group was $75 \%$ versus $6 \%$ in the vehicle-treated group. (10) Thus, E2 administration after major blood loss markedly prolongs permissive hypotension for 3 hrs and increases long-term survival if fluid resuscitation was provided $3 \mathrm{hrs}$ after major blood loss. This hormone may therefore be a novel adjunct for preserving life under conditions in which fluid resuscitation is not possible after major blood loss for an extended period of time. Thus, the use of agents such as $17 \beta$-estradiol, flutamide, DHEA, metoclopramide, prolactin, 4-hydroxy androstenedione, which are readily available clinically, appear to be safe and novel agents for the treatment of cardiovascular and immunological depression following severe blood loss and for decreasing mortality from subsequent sepsis in males and females, and their use should be evaluated in future trauma studies.

\section{ACKNOWLEDGEMENTS}

Supported by USPHS grants RO1 GM37127, RO1 GM39519 and DARAP W911NF-06-1-0219. 


\section{REFERENCES}

1.Choudhry MA, Schwacha MG, Hubbard WJ, Kerby JD, Rue LW, Bland KI, et al. Gender differences in acute response to trauma-hemorrhage. Shock 2005;24 (Suppl 1):101-6.

2.Bone RC. Toward an epidemiology and natural history of SIRS (systemic inflammatory response syndrome). JAMA 1992;268:3452-5.

3.Angele MK, Schwacha MG, Ayala A, Chaudry $H$. Effect of gender and sex hormones on immune responses following shock. Shock 2000;14:81-90.

4. Raju R, Chaudry IH. Sex steroids/receptor antagonist: Their use as adjuncts following trauma-hemorrhage for improving immune/cardiovascular responses and decreasing mortality from subsequent sepsis. Anesth \& Analg 2008;107:159-66.

5.Yu H-P, Chaudry IH. The role of estrogen and receptor agonists in maintaining organ function following trauma-hemorrhage. Shock 2009;31:227-37.

6.George RL, McGwin G Jr, Windham ST, Melton SM, Metzger J, Chaudry IH, et al. Age-related gender differential in outcome following blunt or penetrating trauma. Shock 2003;19:28-32.

7.Frink M, Pape H-C, Griensven MV, Krettek C, Chaudry IH, Hildebrand F. Influence of sex and age on MODS and cytokines after multiple injuries. Shock 2007;27:151-6.

8.Deitch EA, Livingston DH, Lavery RF, Monaghan SF, Bongu A, Machiedo GW. Hormonally active women tolerate shock-trauma better than do men: a prospective study of over 4000 trauma patients. Ann Surg 2007;246:447-453.

9.Adrie C, Azoulay E, Francais A, Clec'h C, Darques L, Schwebel C, et al. Influence of gender on the outcome of severe sepsis - A reappraisal. Chest 2007;132:1786-93

10.Chen JG, Not L, Ng T, Hubbard WJ, Chatham J, Choudhry MA, et al. 17 $\square$-estradiol (E2) administration after major blood loss improves liver ATP, 3-hour survival and also long-term survival following prolonged hypotension (3 hour) and fluid resuscitation. Shock 2006;25 (Suppl 1):11. 